\title{
Extracellular matrix and the implantation cascade in pigs
}

\author{
R. C. Burghardt, ${ }^{1,3}$ J. A. Bowen, ${ }^{1,3}$ G. R. Newton,, 3 \\ and F. W. Bazer ${ }^{2,3}$ \\ Departments of ${ }^{1}$ Veterinary Anatomy \& Public Health, ${ }^{2}$ Animal Science, ${ }^{3}$ Center for Animal \\ Biotechnology, Institute of Biosciences and Technology, Texas A \& M University, College Station, \\ TX 77843, and ${ }^{4}$ Cooperative Agricultural Research Center, Prairie View A \& M University, \\ Prairie View, TX 77446, USA
}

\begin{abstract}
The structural and functional alterations of uterine epithelial cells that permit the apical-apical union of conceptus and uterine epithelium are complex and are likely to involve many different adhesion molecules with distinct but inter-related functions. A number of changes in the molecular composition at the apical surface of uterine epithelial cells associated with the transition from the pre-receptive to the receptive state in the pig uterus are reviewed. Molecules that function in the adhesion cascade resulting in implantation are represented by a variety of adhesion systems. However, integrins are probably the dominant adhesion systems because their capacity to mediate adhesion is linked to their activation by engaging other surface molecules.
\end{abstract}

\section{Introduction}

The process of implantation in pigs follows a prolonged preimplantation period (the pre-receptive stage) upon arrival of the embryo in the uterus. The preimplantation period is characterized by migration, spacing and elongation of conceptuses and secretion of significant amounts of oestrogen, the signal for maternal recognition of pregnancy, during initial elongation (Geisert et al., 1982a,b). The transition to the receptive state is accompanied by structural and functional changes in endometrial surface and glandular epithelial secretions (Bjorkman et al., 1981; Dantzer, 1985; Keys and King, 1990; Stroband and van der Lende, 1990; Roberts et al., 1993). Receptivity is permissive to the uncommon interaction between apical plasma membranes of the genetically distinct endometrial epithelium and embryonic trophectoderm about 7 days after hatching of the embryo from the zona pellucida. Blastocyst invasion of endometrial stroma and decidualization does not occur in pigs; rather the intimate association between uterine epithelium and conceptus tissues is retained (epitheliochorial placentation). Consequently, the trophoblast and its supporting layer of extra-embryonic mesoderm is simply apposed to the uterine epithelium (Fig. 1) and the conceptus remains within the uterine lumen throughout gestation (Burton, 1992). The implantation process is complete with the formation of a placenta as a means to support fetal development to the end of pregnancy.

Although the pig blastocyst does not normally invade the uterine endometrial epithelium during implantation, it will invade and undergo syncytium formation when transplanted to an ectopic site (Samuel and Perry, 1972). The barrier to implantation in the pre-receptive stage and the refractory stage that develops in the absence of pregnancy or failure of pregnancy recognition is a maternal property resident in the uterine epithelium until it is cyclically transformed to respond to embryonic signals and permit contact with conceptus epithelial tissues in the receptive phase. While our understanding of the implantation process in pigs remains incomplete, opportunities to investigate early stages of the process including conceptus-uterine epithelium apposition and adhesion or attachment has the advantage that the absence of conceptus invasion of the 


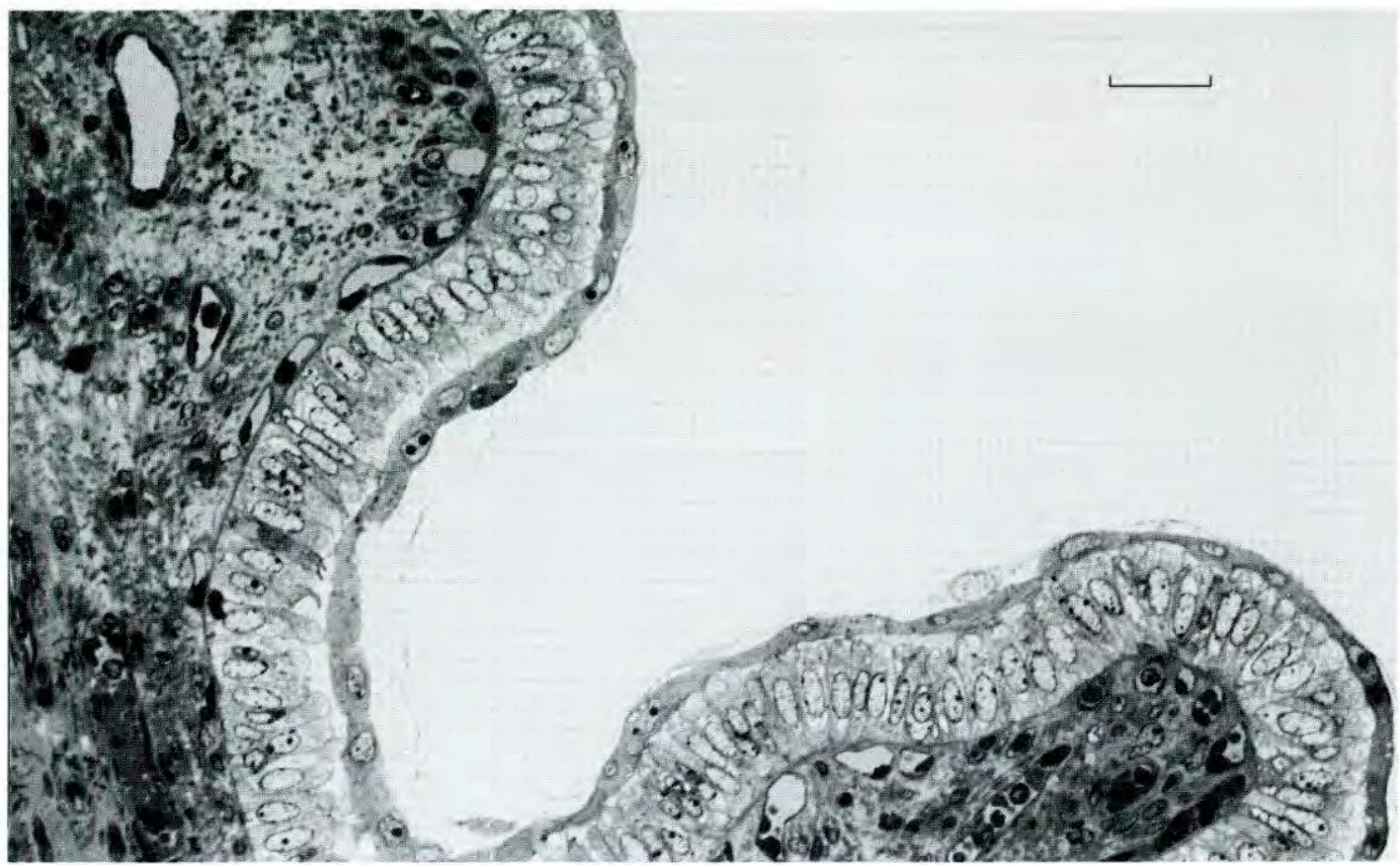

Fig. 1. Light micrograph of conceptus-uterine epithelial interface on day 15 of pregnancy. Scale bar represents $25 \mu \mathrm{m}$.

endometrium (as in haemochorial and endotheliochorial placentation) may not obscure or erase earlier events.

The restricted adhesive properties of a blastocyst to a uterine epithelium that is appropriately prepared by ovarian steroid hormones suggests that, under certain conditions, the apical surfaces of uterine epithelial cells express molecules that do not permit (or prevent) blastocyst attachment. Experimental evidence is accumulating that the uterine epithelium responds to a variety of regulatory agents (growth factors, cytokines) in addition to the action of ovarian steroids that modulate structural and functional properties of the uterine epithelium during the transformation from the non-receptive to the receptive state. Insight into the specialized characteristics of uterine epithelial and stromal cells and their interactions has also provided improvements in culture systems that permit the discrimination of functions that are under direct hormonal control from those that are controlled through hormonal effects on other uterine cell types or more distant tissues. Procedures for culturing uterine epithelium in a polarized fashion (Glasser et al., 1988) have stimulated renewed analysis of epithelial receptivity in vitro. Because polarized epithelial cells regain their non-receptive state in vitro and mimic attributes of these cells in vivo (see Glasser and Mullholland, 1993), the utility of these model systems to support the search for, and analysis of, potential markers of pre-receptive and receptive stages is increasing. This review examines several changes in the molecular composition at the apical surface of uterine epithelial cells that are associated with the transition from the pre-receptive to the receptive state in a variety of species, several of which may be relevant in pigs. Particular attention is directed to endpoints that can be reproduced in polarized endometrial epithelial cells. A consideration of extracellular matrix molecules, their receptors and adhesion systems as potential contributors to the receptive state is also included.

Although the following discussion focuses primarily on the epithelial cell component of the endometrial compartment during the peri-implantation period, the potential contributions of other cells to the generation of the non-receptive and receptive status of the epithelium must be mentioned. Despite the fact that the principal cells of the endometrium are separated by a basal 
lamina, the classic experiments of Cunha et al. (1983) define the importance of epithelial-stromal cell interactions in the production of stage-specific responses of the epithelium to endocrine signals. When the uterus enters the receptive phase, circulating progesterone concentrations are high and these conditions result in a marked decrease in immunologically detectable steroid receptors in the luminal epithelium (Geisert et al., 1993, 1994). Other cells including stroma, myometrium and vascular cells are likely to mediate the action of steroid hormones (for example oestromedins, progestomedins) on the surface epithelium. For example, keratinocyte growth factor (KGF, also known as FGF-7) has been shown to be a stroma-derived, progesterone-induced growth factor that may function as a progestomedin on uterine epithelial cell proliferation in primates (Koji et al., 1994). The selective expression of KGF in stroma and the KGF receptor in uterine epithelial cells has recently been reported in pigs (Liu et al., 1996) and indicates the paracrine function of this growth factor in regulating the properties of pig uterine epithelial cells. Similarly, insulin-like growth factor I (IGF-I) and epidermal growth factor (EGF) may also be mediators of oestrogen action in the uterus (Guidice et al., 1993; Hana and Murphy, 1994).

\section{Pre-receptive Stage}

During the pre-receptive stage, uterine epithelial cells exhibit an abundant negatively charged apical glycocalyx which diminishes during the receptive phase in most mammals (see Denker, 1993). Lectin-binding characteristics of the pig blastocyst surface change just before attachment (Whyte and Robson, 1984) and the glycocalyx of the uterine epithelium is reduced in thickness at the implantation site during initial attachment (Dantzer, 1985; Blair et al., 1991). Although few candidate glycoprotein markers for the pre-receptive stage have been investigated in detail, Muc-1 and CCAM, a member of the immunoglobulin superfamily of glycoproteins (Etzioni, 1996) have been evaluated as potential contributors to maintenance of the pre-receptive state (Svalander et al., 1990; Valdizan et al., 1992; Braga and Gendler, 1993). Of these, only Muc-1 has been investigated in pigs (Bowen et al., 1996a; 1997).

The large (200-1000 kDa) heavily glycosylated integral transmembrane glycoprotein, Muc-1, is predominantly associated with the apical domain of epithelial cells, including uterine epithelium, in a variety of species (Pemberton et al., 1992) and may function as an anti-adhesive molecule in both cadherin- and integrin-mediated cell adhesion events in vitro (Ligtenberg et al., 1992; Wesseling et al., 1995). Muc-1 mRNA and protein are downregulated during the implantation window in rodents (Braga and Gendler, 1993) and thus Muc-1 may function as an anti-adhesive molecule during the peri-implantation period in rodents by masking embryo attachment sites (Surveyor et al., 1995).

Similarly, the expression of Muc-1 during the oestrous cycle and early pregnancy in pigs is consistent with its postulated role as an anti-adhesive molecule (Bowen et al., 1996a). Antibodies directed to the cytoplasmic domain of Muc-1 revealed intense staining on the apical surface of luminal and glandular uterine epithelium on days 0 and 4 . Staining was diminished on day 8 and was not detected on days 10-15 of the oestrous cycle or pregnancy. Therefore, increasing progesterone concentrations correlated with reduced Muc-1 staining in both cyclic and pregnant gilts. These data were further confirmed in ovariectomized gilts treated with exogenous steroids. Immunoreactive Muc-1 on the apical surface of uterine epithelium was present in ovariectomized gilts treated with either vehicle or oestradiol, whereas Muc-1 staining was virtually absent in uterine epithelium of gilts treated with progesterone or oestradiol plus progesterone. These results support the suggestion that reduction or loss of Muc-1 could contribute to the acquisition of endometrial receptivity by exposing constitutively expressed or hormone-induced adhesion molecules or their ligands or both.

The modulation of Muc-1 expression observed in cyclic, pregnant, and ovariectomized gilts is a property that has recently been reproduced in endometrial epithelial cells in vitro (Bowen et al., 1997), if conditions for development and maintenance of epithelial cell polarity (Bowen et al., 1996b) were provided. Uniformly low Muc-1 staining was observed at the apical surface of polarized uterine epithelial cells incubated in medium containing charcoal-stripped fetal bovine serum, 
progesterone, or oestrogen plus progesterone, whereas cells incubated with oestradiol exhibited a marked increase in Muc-1 staining. However, it remains to be determined whether the upregulation of Muc-1 causes polarized epithelial cells to regain their non-receptive state to blastocysts and trophoblastic vesicles in vitro.

\section{Receptive Stage}

The identification of glycoconjugates that may contribute to the receptive state in mammals has been motivated largely by an interest in the development of techniques for assisted human reproduction in infertile couples. The search for markers that identify or predict receptivity to implantation and the development of noninvasive approaches to detect uterine receptivity is ongoing. While comparative animal studies are contributing greatly to an understanding of implantation, details of the maternal-fetal interactions and adhesion systems in epitheliochorial implantation are not as well developed as in species exhibiting invasive implantation.

\section{Integrins and extracellular matrix}

The integrins are a family of glycoconjugates containing members that are expressed on the uterine luminal epithelium and that have spatial and temporal relationships with blastocyst development and implantation. These cation-dependent heterodimeric intrinsic membrane glycoproteins, comprising non-covalently bound $\alpha$ and $\beta$ subunits, participate in cell-cell and cell-substratum adhesion by virtue of their large extracellular domains (Hynes, 1992). At least 23 heterodimer combinations are known to be formed from the currently identified $17 \alpha$ and eight $\beta$ subunits (Luscinskas and Lawler, 1994) with ligand specificity determined by the $\alpha$ and $\beta$ subunit combinations.

Although integrins are expressed on virtually all cells, the spatial and temporal distribution and modulation of several subunits in the human female reproductive tract during the reproductive cycle and early pregnancy have led to the notion that they are directly involved in the process of implantation (Tabibzadeh, 1990; Lessey et al., 1992). The endometrium is presently the only tissue identified that demonstrates hormone-dependent integrin expression (Lessey et al., 1996). The developmental regulation of their expression on the invasive mouse blastocyst during invasion is also consistent with their potential involvement in this process (Sutherland et al., 1993). Their role in adhesion, migration, invasion and a multitude of intracellular effects on organization of the cytoskeleton as well as bi-directional signalling makes integrins attractive as participants in the complex events of implantation. The bi-directional signalling processes (Humphries, 1993) can involve ligand binding through an integrin receptor leading to specific effects on cytoskeletal organization, activation of signalling pathways, and to the eventual regulation of gene expression (i.e. outside-in signalling). In addition, integrin cytoplasmic domains transduce cell type-specific signals that modulate ligand binding affinity (i.e., inside-out signalling). The involvement of these central functions of integrin receptors in trophectoderm-uterine epithelial cell interactions has been suggested by Damsky et al. (1993).

Much of the data on uterine integrin receptors have come from clinical studies and experiments in rodents; only recently have analyses been conducted in an epitheliochorial animal model (Bowen et al., 1996a). Therefore, comparative information relative to invasive and non-invasive modes of implantation is only beginning to emerge. At least 14 different integrin subunits (nine $\alpha$ and five $\beta$ integrin subunits) have been identified in human endometrium (Lessey et al., 1996) and seven subunits (five $\alpha$ and two $\beta$ integrin subunits) in the pig uterine endometrium to date (Bowen et al., 1996a). The uterine epithelial cell expression of several integrin subunits in both species is temporally and spatially regulated during the reproductive (oestrus or menstrual) cycle while others are constitutively expressed. The species-specific differences may reflect the major differences in the mode of implantation, although several common subunits have been identified. 

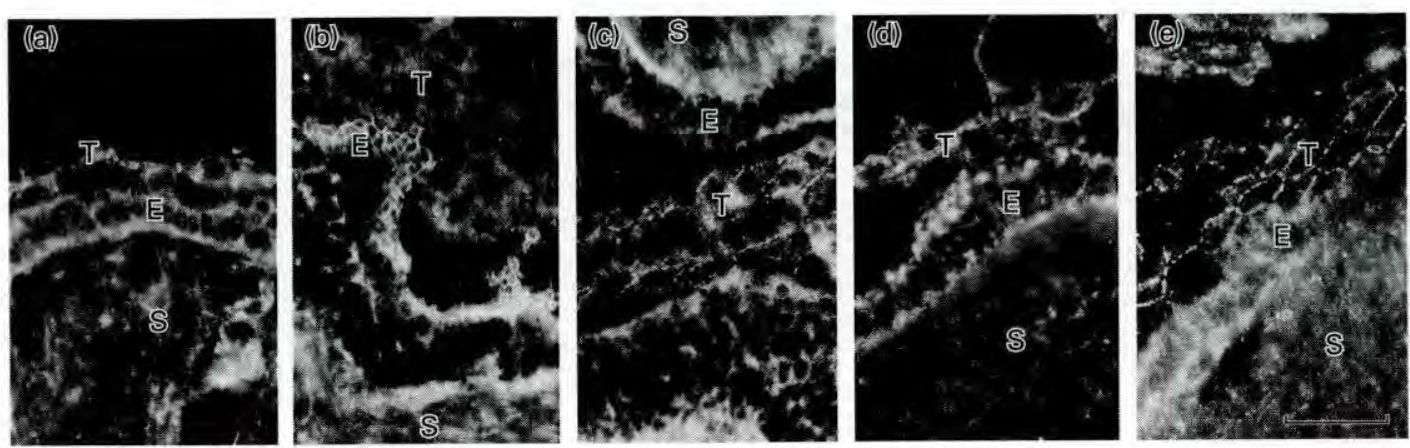

Fig. 2. Immunofluorescence analysis of integrin subunit expression at implantation sites at day 15 . Individual subunits shown include (a) $\alpha 4$, (b) $\alpha 5$, (c) $\alpha \mathrm{v}$, (d) $\beta 1$ and (e) $\beta 3$ staining in stroma (S), uterine epithelium (E) and trophectoderm $(\mathrm{T})$. Scale bar represents $50 \mu \mathrm{m}$.

In humans, considerable attention has been given to $\alpha v \beta 3$ as a marker of uterine receptivity (Lessey et al., 1992, 1996). The expression of $\alpha v \beta 3$, which was localized along apical microvilli and secretory granules of endometrial epithelial cells, is regulated during the menstrual cycle with maximal expression coincident with the rise in progesterone during the period of maternal recognition of pregnancy; aberrant expression of $\alpha v \beta 3$ is associated with infertility (Lessey et al., 1996).

Pig uterine luminal epithelial cell expression of integrin subunits $\alpha 1, \alpha 3, \alpha 4, \alpha 5, \alpha v, \beta 1$ and $\beta 3$, monitored during the oestrous cycle and early pregnancy (including sites of conceptus attachment) (Bowen et al., 1996a), revealed three distinct patterns of expression. These patterns were classified as: (1) low or no expression ( $\alpha 1$ and $\alpha 3$ ); (2) high intensity constitutive expression ( $\alpha$ v and $\beta 3$ ); and (3) high intensity, modulated expression $(\alpha 4, \alpha 5, \beta 1)$. Modulation of $\alpha 4, \alpha 5, \beta 1$ resulted in maximal expression during the period corresponding to maternal recognition of pregnancy (i.e. days 10 to 15). In the same study, experimental evidence for steroid modulation of integrin subunits was obtained in ovariectomized gilts. Integrin subunits classified as the high intensity, modulated subunits $(\alpha 4, \alpha 5, \beta 1)$ were expressed at a higher intensity in gilts that were treated with progesterone (or progesterone in combination with oestrogen) than in oestrogen-treated animals.

Analysis of implantation sites indicated that integrin subunits $\alpha 4, \alpha 5, \alpha \mathrm{v}, \beta 1$ and $\beta 3$ were expressed at sites of trophectoderm contact with the uterine epithelium (Fig. 2). The integrin receptors potentially formed from the heterodimers identified in the study included $\alpha 4 \beta 1, \alpha 5 \beta 1$, $\alpha v \beta 1$ and $\alpha v \beta 3$. These receptors, like those identified in human studies during the initial events of attachment are all members of the fibronectin-vitronectin family of integrin receptors (Hynes, 1992).

The pig conceptus was also found to express the same integrin subunits $(\alpha 4, \alpha 5, \alpha v, \beta 1$ and $\beta 3)$ as the uterine epithelium. The significance of the integrin receptors and their modulation must be considered in the context of the available receptors and ligands present on both conceptus and uterine epithelial cells. Apical expression of the extracellular matrix constituents fibronectin and vitronectin is present on the pig conceptus, whereas vitronectin is present on uterine epithelium during early implantation (Fig. 3) (Bowen et al., 1996a). Human studies suggest the involvement of fibronectin in mediating attachment, trophoblast spreading and syncytial formation (Kao et al., 1988; Kliman and Feinberg 1990; Bischof et al., 1995).

Further characterization of the nature of the fibronectin in the pig conceptus and uterus has revealed the constitutive expression of oncofetal fibronectin by both pig conceptus and uterus throughout gestation (Tuo and Bazer, 1996). This fibronectin variant, which exhibits two distinct epitopes, one of which is created by glycosylation of a single amino acid, is absent from normal adult tissues (see Feinberg et al., 1995). The presence of oncofetal fibronectin and vitronectin at the fetal-maternal interface suggests that it plays a role in conceptus interactions, attachment to the endometrium or both processes. This interpretation is strengthened by the fact that the distribution and type of ECM components correspond to the integrin subunits expressed on uterine epithelial 

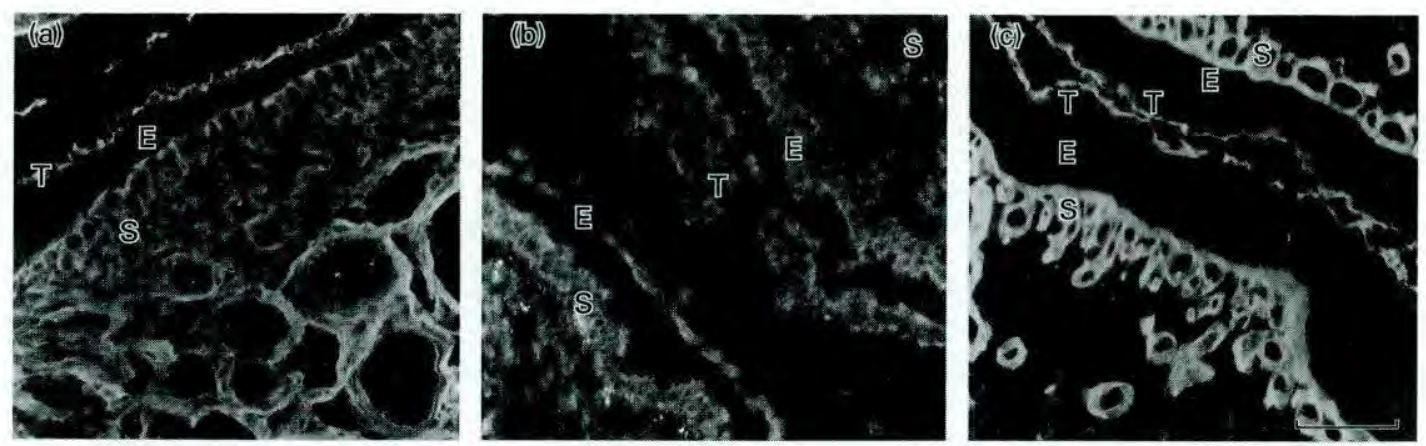

Fig. 3. Immunofluorescence analysis of extracellular matrix expression at implantation sites at day 15 . Staining of (a) fibronectin, (b) vitronectin and (c) type IV collagen are shown. Stroma (S), uterine epithelium (E) and trophectoderm (T) are identified. Fibronectin was present on trophectoderm but not epithelial cells whereas vitronectin was present on both. Type IV collagen staining was restricted to basal lamina of epithelium and trophectoderm and was common in stromal vessels. Scale bar represents $50 \mu \mathrm{m}$.

cells during implantation in pigs. It is also relevant that in human trophoblasts, transforming growth factor- $\beta$ (TGF- $\beta$ ) stimulates oncofetal fibronectin synthesis (Feinberg et al., 1994) and three TGF- $\beta$ isoforms (TGF- $\beta 1$, TGF- $\beta 2$, and TGF- $\beta 3$ ) and type I and II receptors have been localized in periimplantation pig conceptuses (Gupta et al., 1996).

Integrins $\alpha 1 \beta 1$ and $\alpha 3 \beta 1$ are members of the collagen-laminin receptor family. The restricted expression of these integrins at the basal aspect of pig uterine epithelium is consistent with cellular attachment to the basement membrane and their unlikely involvement in interactions between the trophectoderm and uterine epithelium. Integrins $\alpha 4 \beta 1, \alpha 5 \beta 1$ and $\alpha v \beta 1$ are members of the large family of fibronectin receptors, which makes them possible candidates for trophectoderm-uterine epithelium attachment based upon the finding that fibronectin and vitronectin are present on the contacting surfaces between the conceptus and the uterine epithelium. In addition to its ability to bind vitronectin, $\alpha v \beta 3$ is capable of binding to oncofetal fibronectin and osteopontin. The expression of several different fibronectin receptors on the uterine epithelium and the trophectoderm allows the integrins to bind to fibronectin at two different locations, $\alpha 4 \beta 1$ integrin binds to the amino acid sequence, EILDV (Glu-Ile-Leu-Asp-Val), within the IIICS region of fibronectin, while the remaining integrins will bind to the RGD sequence of fibronectin (Hynes, 1992). The $\alpha v \beta 1$ (weak affinity vitronectin receptor) and $\alpha v \beta 3$ (vitronectin receptor) can also bind to the RGD sequence found in fibronectin. Thus, the four integrin heterodimers and two ECM proteins (fibronectin and vitronectin) are available for participation in implantation events of the pig conceptus. Although not yet described in pigs, osteopontin has been shown to be a ligand for $\alpha v \beta 3$ in primate endometrium (Fazleabas et al., 1997).

The redundancy in integrin receptors may ensure the ability of the conceptus to attach to the uterine epithelium. However, it is possible that each of these integrins may carry out different functions related to attachment and cell signalling (Meredith et al., 1996). For example, $\alpha 5 \beta 1$ interacts with cytoskeleton, mediates cell spreading on fibronectin, and plays a role in matrix assembly (Lafrenie and Yamada, 1996). In addition to providing a direct link between ECM and cytoskeleton, these integrins also regulate the production of second messengers within cells. A common feature of integrin-dependent cell spreading or integrin receptor clustering is tyrosine phosphorylation of cytoplasmic proteins and the activation of the $\mathrm{Na}^{+}-\mathrm{H}^{+}$antiporter, producing high intracellular $\mathrm{pH}$ (Schwartz et al., 1991). However, the integrin-dependent adhesion and spreading that results in intracellular $\mathrm{Ca}^{2+}$ spikes and oscillations (Schwartz, 1993) seems to be specific for a particular integrin ( $\alpha v \beta 3$ ) (Schwartz and and Denninghoff, 1994). These observations suggest that different receptors within the same cell can generate unique signals. Integrin ligand binding and aggregation leads to a transmembrane hierarchy of molecular responses characterized by the prompt transmembrane accumulation of a large class of at least eight cytoskeletal and over 20 signal 
transduction molecules, and upregulation of cytoplasmic protein tyrosine phosphorylation (Miyamoto et al., 1995). Among the signalling molecules are focal adhesion kinase (pp125 $5^{\mathrm{AAK}}$ ), c-Src, cFyn, RhoA, Rac1, Ras, and several MAP kinases.

Regardless of the mode of implantation, it appears that the expression of integrin receptors and their ligands are important elements of adhesion, attachment and cell signalling between the conceptus and uterine epithelium. Integrin receptors expressed on the conceptus appear to be largely conserved among species examined to date, and consist of integrins that are also expressed on the uterine epithelium. This strategy may represent a conserved mechanism of conceptus attachment. The induction of new integrin receptor populations (specific for collagen and laminin) in species that exhibit invasive implantation occurs later in conceptus development (Sutherland et al., 1993). Evaluation of the functional roles of the integrins expressed on pig uterine epithelium and trophectoderm should provide important insights into the relationship of integrin ligation and signal transduction during adhesion and attachment stages of early implantation with broad relevance to reproductive efficiency in a variety of species.

Analysis of integrin expression in pig uterine epithelial cells and conceptus-derived trophoblastic vesicles in vitro suggests that the above referenced integrin subunits identified on conceptus trophectoderm and in uterine epithelium of cyclic and pregnant gilts are expressed in vitro (Bowen et al., 1997). However, comparable modulation of integrin subunits by steroid hormones was detected in uterine epithelial cells only if conditions for development and maintenance of epithelial cell polarity were provided. Although polarized uterine epithelial cells and trophoblastic vesicle culture systems require further refinements, they may prove useful as in vitro model systems to study conceptus-endometrial interactions during the peri-implantation period and the control of uterine receptivity. At this time, it is not feasible to search for direct uterine epithelial cell-trophectoderm signalling in vivo; therefore culture systems that express the hormonally modulated adhesion systems observed in vivo provide opportunities for investigating integrinmediated signal transduction in pigs.

\section{pGP30 and IaIH4}

Another glycoprotein the expression of which is correlated with uterine receptivity in pigs is a 30 $\mathrm{kDa}$, basic endometrial glycoprotein (pGP30). Initial antibody studies showed that pGP30 expression changes during the oestrous cycle and early pregnancy (Geisert et al., 1995). Maximal staining intensity was observed in surface epithelium on day 12 of the oestrous cycle and from days 12 to 18 of pregnancy; the appearance of pGP30 was stimulated by progesterone administration to ovariectomized gilts. Staining in the area of trophoblast attachment suggested that the glycoprotein is available to the conceptus at the uterine luminal interface. Conceptus oestrogen secretion appears to alter pGP30 secretion from endometrial cultures (Geisert et al., 1995).

More recent analysis has indicated that pGP30 is a cleavage product of a larger pig $120 \mathrm{kDa}$ multi-functional inter-trypsin inhibitor heavy chain-like (I $\alpha \mathrm{IH} 4)$ protein (Geisert et al., 1996). It appears, therefore, that pGP30 may be a useful marker of receptivity, while I $\alpha \mathrm{IH} 4$ may be the functionally relevant player in conceptus-epithelial interaction. The unique properties of pig I $\alpha \mathrm{IH} 4$, its interaction with the extracellular matrix and the potential roles of pig I $\alpha \mathrm{IH} 4$ in the initial stages of trophoblastic attachment and regulation of trophoblast invasiveness are detailed by Geisert and Yelich (this supplement). Noteworthy is the observation that all $I \alpha I$ heavy chains contain a von Willebrand type A domain, a recognition site for the $\alpha v \beta 3$ integrin receptor and other adhesion molecules.

\section{LNF and related determinants}

Changes in carbohydrate expression on the epithelium lining the uterine lumen were initially suggested by the reduction in surface charge and thinning of the glycocalyx near the time of implantation (Enders and Schlafke, 1974). Subsequent studies in rodents identified a specific 
oligosaccharide carrying an antigen at its nonreducing terminal carbohydrate determinants which may play a role in the implantation process. Monoclonal antibodies developed against a blood group determinant, H-type-1 antigen, carrying the lacto- $N$-fucopentaose- 1 epitope (LNF-I, Fuc $\alpha 1$ 2 Gal $\beta 1-3 G$ lcNAc $\beta 1-)$ detected changes in expression of this epitope on the surface of mouse uterine epithelial cells during the pre-implantation period (Kimber et al., 1988; Lindenberg et al., 1988; Kimber and Lindenberg, 1990). LNF-I (but not polysaccharides with similar structures such as LNFII, LNF-III, lacto- $N$-difucohexaose, lacto- $N$-tetraose, and lactodifucotetraose) (Kimber et al., 1994) and an anti-LNF-I monoclonal antibody were also shown to inhibit mouse embryo attachment significantly using an in vitro model for implantation (Lindenberg et al., 1988).

In ovariectomized mice, the LNF-I epitope appeared to be stimulated by oestrogen (Kimber and Lindenberg, 1990) rather than progesterone as in rats (Kimber et al., 1995). In pregnant mice, maximal LNF-I epithelial surface expression occurred on days 1-4 and decreased markedly by days 5 and 6 (Kimber et al., 1988). Since the expression of LNF-I does not span the entire duration of the receptive phase, the potential role for LNF-I may be limited to initial steps of adhesion. Mouse conceptuses are believed to contain specific binding sites for LNF-I, although specific receptors have not been identified.

LNF-I expression in pigs has recently been examined using the antibody used by Kimber $e$ al. $(1994,1995)$ in mouse and rat. Expression was greatest in cyclic gilts from days 0-4 and is weak on days 8-15 (Fig. 4). In ovariectomized gilts, LNF-I was detected in oestradiol- but not in progesterone, oestradiol plus progesterone-, or vehicle-treated animals (Fig. 5). Analysis of the LNF-I expression in the polarized uterine epithelial culture system is in progress.

Another carbohydrate antigen localized by immunostaining on luminal epithelium of pregnant pigs from day 14 to day 30 of pregnancy which was specifically blocked by LNF-III is the Lewis ${ }^{x}$ (Galß-1[Fuc $\alpha 1-3]$ GlcNAc $\beta 1-)$ determinant (Whyte and Binns, 1994). The oligosaccharide carrying this antigen at its nonreducing terminal can function as a ligand for E-selectin and has also been shown to play a role in homotypic adhesion $\left(\right.$ Lewis $^{\mathrm{x}}-$ Lewis $^{\mathrm{x}}$ ) in mouse compaction and F9 embryonal carcinoma cell adhesion (Hakomori, 1992). The absence of immunoreactive Lewis ${ }^{x}$ on pig trophectoderm indicates the absence of a role for Lewis ${ }^{x}$ homotypic adhesion in pig uterine epithelial cell-trophectodermal interaction (Whyte and Binns, 1994).

\section{Selectins}

The above referenced identification of Lewis ${ }^{x}$ on pig uterine epithelium has led to the search for the expression of selectins on uterine epithelial cells and trophectoderm. Selectin family members include E-, P-, and L-selectin. The term selectin refers to the common amino-terminal lectin domain and the selective function and expression of these molecules; the nomenclature is based upon the initial detection in cells (i.e. endothelium, platelet and leukocyte, respectively). All members of the selectin family contain the unique extracellular region composed of an amino-terminal calciumdependent lectin domain, which is central to the ability of each of the selectins to bind the Lewis ${ }^{x}$ antigen (Tedder et al., 1995; Etzioni, 1996).

Neither E-selectin nor L-selectin were detected within pig uterine endometrial tissue or on trophectoderm by immunocytochemistry, whereas E-selectin was detected in inflammatory cutaneous sites and L-selectin in high endothelial venule cells in maternal lymph nodes in these pigs (Whyte and Binns, 1994). The significance of Lewis ${ }^{x}$ and LNF-I expression in uterine epithelium and the possible involvement of carbohydrate-selectin interactions in trophoblast-uterine epithelial cell adhesion remains undefined.

\section{HSPGs and HSPG-binding proteins}

A body of evidence suggests that heparan sulfate proteoglycans (HSPG) support rodent blastocyst interactions with a variety of substrates including uterine epithelial cells during early attachment (see Wegner and Carson, 1994). HSPGs are developmentally regulated at the surface of 

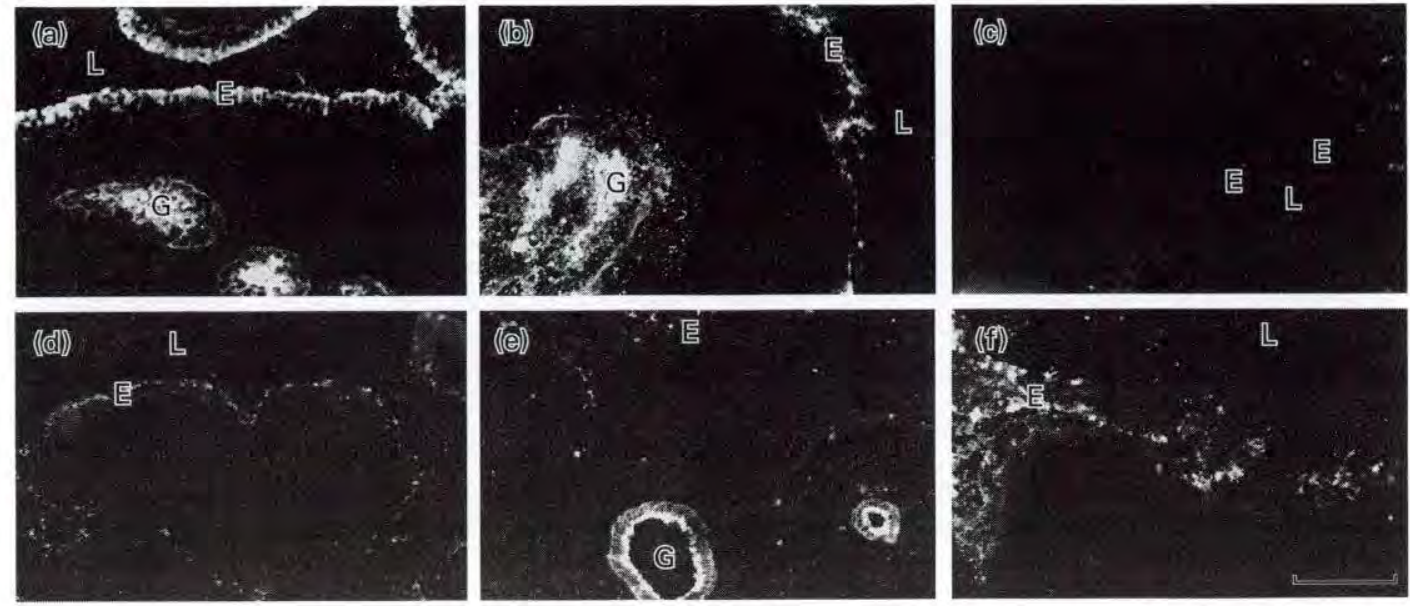

Fig. 4. Immunofluorescence analysis of uterine epithelial lacto- $N$-fucopentaose I (LNF-I) expression in cyclic gilts on days (a)0, (b)4, (c)8, and on days (d)11, (e)13 and (f) 15 on luminal epithelial cells (E) and glands (G). The lumen of the uterus (L), where present, is identified in each panel. LNF-I expression was greatest in cyclic gilts from days $0-4$ and is weak on days $8-15$. Scale bar represents $50 \mu \mathrm{m}$.

mouse blastocysts and are detected at sites of embryo-uterine epithelial cell attachment and at the surface of human trophoblastic cell lines (Wegner and Carson, 1994). HSPGs support adhesive interactions of numerous cell types and extracellular matrix constituents and are capable of complexing with a number of growth factors and modulate cellular functions. A heparin-binding epidermal growth factor-like growth factor (HB-EGF) is synthesized as a transmembrane form in mouse uterine epithelium. The mRNA encoding HB-EGF is expressed in uterine luminal epithelium just before implantation and only at the site of blastocyst apposition, and experimental evidence has been provided that HB-EGF can function as a juxtacrine adhesion factor that could be one of the mediators of blastocyst adhesion during early implantation (Raab et al., 1996).

Although comparable analyses of HSPG expression in pig conceptus and uterine epithelial cells have not yet been conducted, the uterine expression of colony-stimulating factor 1 (CSF-1) in the pig exhibits an interesting parallel to HB-EGF. CSF-1 has been detected on the apical side of pig trophectoderm and immunocytochemical analysis suggests that this may be a membrane-bound form of CSF-1 (Tuo et al., 1995). CSF-1 may interact with CSF-1 receptor expressed on both trophectoderm and endometrial cells by a juxtacrine mechanism as the CSF-1 receptor is expressed by both pig conceptus and endometrium (W. Tuo and F.W. Bazer, unpublished).

\section{Implantation as an Adhesion Cascade}

Adhesion events that occur during implantation involve a highly coordinated set of sequential interactions that may involve many different adhesion molecules with distinct but inter-related functions. The molecular interactions involved in apposition and attachment of the conceptus and apical uterine luminal epithelial cell surface may constitute an implantation adhesion cascade with core events comparable to other adhesion cascades (Fig. 6). Examples include the cytokine-mediated activation of granulocyte-endothelial interactions and metastasis of malignant cells (see Schweighoffer and Shaw, 1992; Giancotti and Mainiero, 1994). Similarities of early implantation events to the leukocyte-endothelial adhesion cascade and metastatic process have been recognized (Damsky et al., 1993; Kimber et al., 1994).

Several core elements of adhesion cascades have been described (Schweighoffer and Shaw, 1992) including (1) a triggering molecule on the cell surface capable of interacting with an extracellular ligand, (2) a biochemical signal (or series of signals) generated by physical association of the trigger 

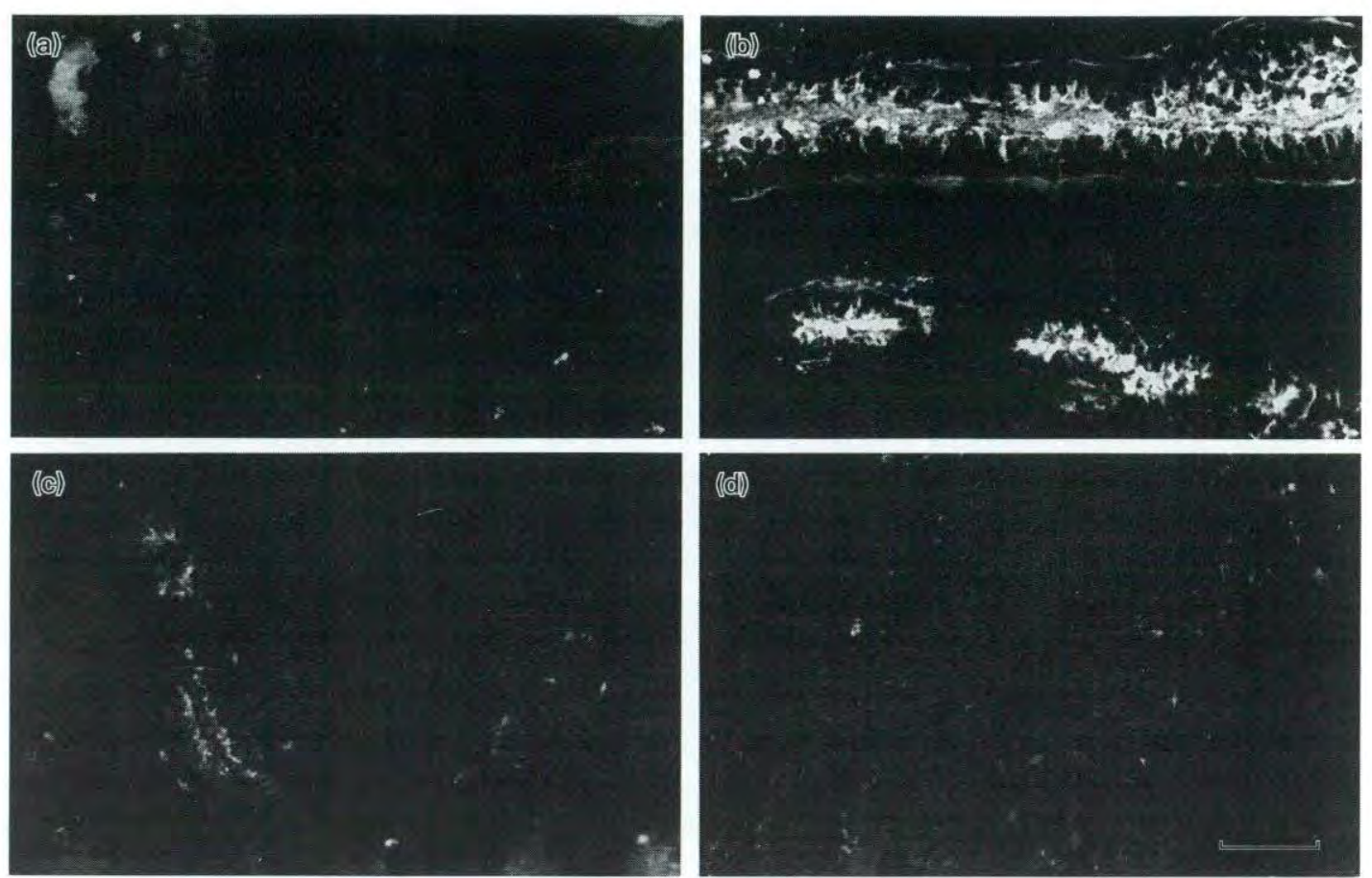

Fig. 5. Immunofluorescence analysis of uterine epithelial lacto- $N$-fucopentaose (LNF-I) expression in ovariectomized gilts receiving vehicle carrier (a), oestradiol (b), progesterone (c) or oestradiol plus progesterone (d). Daily doses of oestradiol valerate $\left(100 \mu \mathrm{g} \mathrm{day}^{-1}\right)$, progesterone $\left(200 \mathrm{mg} \mathrm{day}^{-1}\right)$, or oestradiol plus progesterone were administered for 10 days. LNF-I staining was intense only in oestradiol-treated gilts. Scale bar represents $50 \mu \mathrm{m}$.

molecule to the adhesion molecule, and (3) an adhesion molecule, the adhesive nature of which is altered by the biochemical signal. The molecules that function to trigger the adhesion cascade are diverse and represented by many families of molecules that include cytokine receptors, carbohydrate-selectin interactions, and membrane lipid metabolites that are linked to a variety of signal transduction pathways (Schwartz et al., 1995; Stewart et al., 1995). While there is considerable diversity among triggering molecules, integrins are the dominant adhesion systems in each of the adhesion cascades and their capacity to mediate adhesion is linked to their activation by engaging another surface molecule (Schweighoffer and Shaw, 1992).

The conditions that control the developmental expression of adhesion receptors and ligands in the uterus are regulated precisely through the action of ovarian steroids, growth factors and cytokines. During the pre-receptive phase, the blastocyst is free to move within the uterus, a condition that appears to be supported in a number of species by the non-adhesive nature of the large glycoprotein Muc-1. Muc-1 may extend outward from the apical cell surface further than receptors or triggering molecules and thereby have the capacity to sterically inhibit the interaction of the embryo with adhesion molecules at the maternal-fetal interface (Aplin et al., 1996).

Entry of the uterus into the receptive phase in synchrony with conceptus development and the expression or unmasking of adhesion molecules permits the primary interactions involving lowaffinity bonds with triggering molecules. Several candidates for this role have been described in this review including the pig I $\alpha$ IH4 (see Geisert and Yellich, this supplement), and membrane-associated CSF-1 (Tuo et al., 1995).

Although not discussed in this review, cytokines, growth factors and paracrine signals from trophectoderm may also act as triggering mechanisms or signals involved in the implantation cascade. Potential participants in this process include, but are not limited to, transforming growth 


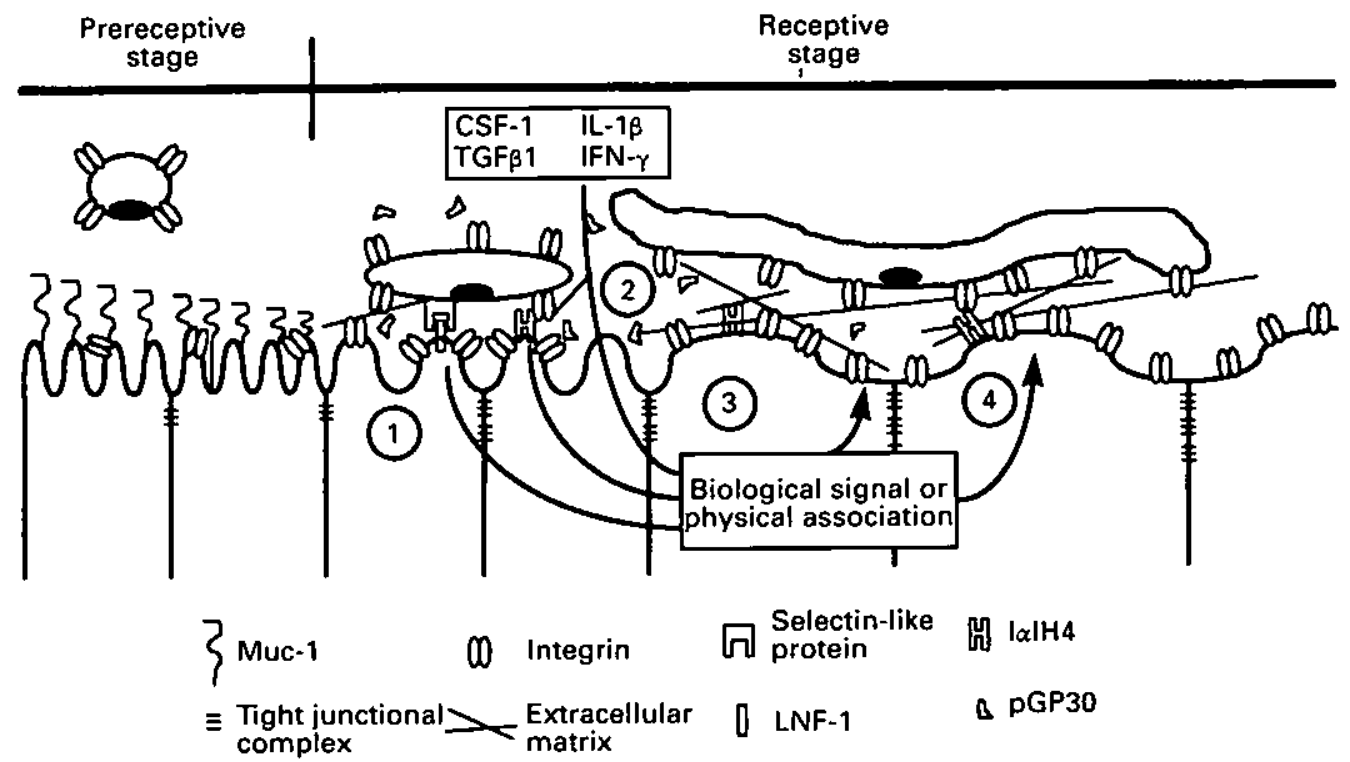

Fig. 6. Implantation as an adhesion cascade. Transition of uterine luminal epithelium from pre-receptive to receptive requires the downregulation of the anti-adhesive mucin, Muc-1, thought to inhibit the interaction of the embryo with adhesion molecules at the interface of the blastocyst and uterine epithelium. The sequential events of implantation (1-4) resemble events of other adhesion cascades. (1) The progesterone-induced structural changes resulting in reduction of epithelial polarity is indicated by loss of apical microvilli, alteration of tight junction organization, and close apposition of conceptus and apical uterine epithelium. (2) Initial interaction between the two genetically distinct cell types occurs with triggering molecules that are either induced or constitutively expressed but unavailable until loss of Muc-1. The triggering molecules that initiate the implantation cascade are involved in low affinity and weak molecular interactions. (3) Transduction of signals from the triggering molecules can include a diverse array of biochemical and physical interactions, including $\mathrm{Ca}^{2+}$ fluxes, protein phosphorylation, or cytoskeletal alterations. (4) The final adhesion event is mediated by the high-affinity integrin-ligand interactions. Potential integrin heterodimers that are candidates for the implantation cascade are all members of the fibronectin and vitronectin family of receptors. LNF-1: lacto- $N$-fucopentaose; I $\alpha$ IH4: inter-trypsin inhibitor heavy chain-like protein; GM-CSF: granulocyte-macrophage colony-stimulating

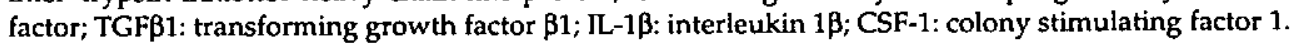

factor $\beta 1$ (TGF- $\beta_{1}$ ), interferon gamma (IFN- $\gamma$ ), granulocyte-macrophage colony-stimulating factor (GM-CSF), and interleukin $1 \beta$ (IL-1B) (Robertson et al., 1994), all of which are secreted by pig trophectoderm during the peri-implantation period. These and other cytokines could initiate integrin activation during signalling events.

It should also be noted that changes in the polarized functions of uterine epithelium that occur during the receptive phase result in cytoskeletal alterations and changes in the distribution of plasma membrane proteins and lipids partitioned by tight junctions. These changes may contribute to conformational alterations in the integrins which could lead to their activation (Conforti et al., 1990).

As discussed previously, the involvement of integrins in adhesion, migration, and invasion, and their numerous effects on the organization of the cytoskeleton and bi-directional signalling are consistent with these adhesion molecules as dominant participants in the implantation cascade. The upregulation of several integrin subunits and exposure of others upon loss of Muc-1 during the receptive period provide a number of heterodimer combinations that are members of the fibronectin and vitronectin family of integrin receptors. The redundancy in the potential integrin receptors present and the variety of potential triggering molecules and ligands highlight the physiological complexity of the implantation cascade in mammals. 
Supported by USDA grants 92-37203-7952 (RCB), 95-37203-2185 (FWB) and NIH grants HD32534 (FWB) $2 \mathrm{~S} 06 \mathrm{GM} 08094$ (GRN). The authors thank S. R. Glasser, L. A. Jaeger and R. D. Geisert for helpful discussions.

\section{References}

Aplin JD, Hey NA and Li TC (1996) MUC1 as a cell surface and secretory component of endometrial epithelium: reduced levels in recurrent miscarriage American Journal of Reproductive Immunology 35 261-266

Bischof P, Haenggeli L and Campana A (1995) Gelatinase and oncofetal fibronectin secretion is dependent on wintergreen expression on human cytotrophoblasts Human Reproduction 10 734-742

Bjorkman N, Dantzer V, Hasselager E, Holm $\mathbf{H}$ and Kjaersgaard $\mathbf{P}$ (1981) Perfusion in vivo of the porcine placenta. Fixation for EM Placenta 2 287-302

Blair RM, Geisert RD, Zavy MT, Yellin T, Fulton RW and Short EC (1991) Endometrial surface and secretory alterations associated with embryonic mortality in gilts administered estradiol valerate on days 9 and 10 of gestation Biology of Reproduction 44 1063-1079

Bowen JA, Newton GR, Weiss DW, Bazer FW and Burghardt RC (1996a) Characterization of a polarized porcine uterine epithelial cell model system Biology of Reproduction 55 613-619

Bowen JA, Bazer FW and Burghardt RC (1996b) Spatial and temporal analyses of integrin and Muc-1 expression in porcine uterine epithelium and trophectoderm in vivo. Biology of Reproduction 55 1098-1106

Bowen JA, Bazer FW and Burghardt RC (1997) Spatial and temporal analyses of integrin and Muc-1 expression in porcine uterine epithelium and trophectoderm in vitro. Biology of Reproduction 56 409-415

Braga VM and Gendler SJ (1993) Modulation of Muc-1 mucin expression in the mouse uterus during the estrus cycle and early pregnancy fournal of Cell Science 105 397-405

Burton GJ (1992) Human and animal models: limitations and comparisons. In The First Thelve Weeks of Gestation pp 469-485 Ed. ER Barnea J Hustin and E Janiaux Springer-Verlag, Berlin

Conforti C, Zanetti A, Paxquali-Ronchetti I, Quaglino D, Neyroz P and Dejana E (1990) Modulation of vitronectin receptor binding by membrane lipid composition Journal of Biological Chemistry $2654011-4019$

Cunha GR, Chung LWK, Shannon JM, Tagichi $O$ and Fujii H (1983) Hormone-induced morphogenesis and growth: role of mesenchymal-epithelial interactions Recent Progress in Hormone Research $39559-598$

Damsky C, Sutherland A and Fisher S (1993) Extracellular matrix 5: adhesive interactions in early mammalian embryogenesis, implantation and placentation FASEB Journal 7 1320-1329

Dantzer V (1985) Electron microscopy of the initial stages of placentation in the pig Anatomy \& Embryology 172 281-293

Denker H-W (1993) Implantation: a cell biological paradox Joumal of Experimental Zoology 266 541-558

Enders AC and Schlafke S (1974) Surface coats of the mouse blastocyst and uterus during the preimplantation period Anatomical Record 180 31-45

Etzioni A (1996) Adhesion molecules - their role in health and disease Pediatric Research 39 191-198

Fazleabas AT, Bell SC, Flemming A, Sun J and Lessey B (1997) Distribution of integrins and the extracellular matrix proteins in the baboon endometrium during the menstrual cycle and early pregnancy Biology of Reproduction 56 348-356

Feinberg RF, Kliman HJ and Wang C-L (1994) Transforming growth factor- $\beta$ stimulates trophoblast oncofetal fibronectin synthesis in vitro: implications for trophoblast implantation in viwo. Joumal of Clinical Endacrinology and Metabolism 78 1241-1248

Feinberg RF, Kliman HJ, Bedian V, Monzon-Bordonaba F, Menzin A $W$ and Wang C.L (1995) Monoclonal antibody X18A4 identifies an oncofetal fibronectin epitope distinct from the FDC- 6 binding site American Joumal of Obstetrics $\&$ Gymecology 172 1526-1536

Geisert RD, Renegar RH, Thatcher WW, Roberts RM and Bazer FW (1982a) Establishment of pregnancy in the pig: I. Internelationships between preimplantation development of the pig blastocyst and uterine endometrial secretions Biology of Reproduction 27 925-940

Geisert RD, Brookbank JW, Roberts RM and Bazer FW (1982b) Establishment of pregnancy in the pig: II. Cellular remodeling of the porcine blastocyst during elongation on day 12 of pregnancy Biology of Reproduction 27 941-955

Geisert RD, Brenner RM, Moffatt RJ, Harney JP, Yellin T and Bazer FW (1993) Changes in oestrogen receptor protein, mRNA expression and localization in the endometrium of cyclic and pregnant gilts Reproduction, Fertility and Development 5 247-260

Geisert RD, Pratt TN, Bazer FW, Mayes JS and Watson GH (1994) Immunocytochemical localization and changes in endometrial progestin receptor protein during the porcine oestrous cycle and early pregnancy Reproduction, Fertility and Development 6 749-760

Geisert RD, Mendan JD, Pratt T, Schmilt RAM, Lessey BA and McCann JP (1995) Isolation and characterization of a 30 $\mathrm{kDa}$ endometrial glycoprotein synthesized during the estrous cycle and early pregnancy of the pig Biology of Reproduction 53 1038-1050

Geisert RD, Yelich JV, Pratt T and Pomp D (1996) Identification of porcine GP30 as inter-trypsin inhibitor family heavy chainrelated protein Biology of Reproduction 54 (Supplement 1) 78a

Giancotti FG and Mainiero F (1994) Integrin-mediated adhesion and signaling in tumorigenesis Biochimica et Biophysica Acta 1198 47-64

Glasser SR and Mullholland J (1993) Receptivity is a polarity dependent special function of hormonally regulated uterine epithelial cells Microscopy Research and Techniques 25 106-120

Glasser SR, Julian J, Decker GL, Tang J-P and Carson DD (1988) Development of morphological and functional polarity in primary cultures of immature rat uterine epithelial cells Joumal of Cell Biology 107 2409-2423

Guidice LC, Dsupin BA, Jin IH, Vu TH and Hoffman AR (1993) Differential expression of messenger ribonucleic acids encoding insulin-like growth factors and their receptors in human uterine endometrium and decidua journal of Clinical Endocrinology and Metabolism 76 1115-1122

Gupta A, Bazer FW and Jaeger LA (1996) Differential expression of beta transforming growth factors (TCF- $\beta 1$, TCF- $\beta 2$, and 
TGF-B3) and their receptors (type I and II ) in periimplantation porcine conceptuses Biology of Reproduction 55 796-802

Hana V and Murphy LJ (1994) Intendependence of epidermal growth factor and insulin-like growth factor-l expression in the mouse uterus Endocrinology 135 107-112

Hakomori S (1992) Le $\mathrm{Le}^{\star}$ and related structures as adhesion molecules Histochemical Journal 24 771-776

Humphries MJ (1993) Mechanisms of ligand binding by integrins Biochemical Society Transactions 22 275-282

Hynes RO (1992) Integrins: versatility, modulation, and signaling in cell adhesion Cell 69 11-25

Kao LC, Caltabiano S, Wu S, Strauus JF and Kilman H (1988) The human villous cytotrophoblast: interactions with extracellular matrix proteins, endocrine function, and cytoplasmic differentiation in the absence of syncytium formation Developmental Biology 130 639-702

Keys JL and King GJ (1990) Microscopic examination of porcine conceptus-maternal interface between days 10 and 19 of pregnancy American Journal of Anatomy 188 221-238

Kimber SJ and Lindenberg S (1990) Hormonal control of a carbohydrate epitope involved in implantation in mice Joumal of Reproduction and Fertility 89 13-21

Kimber SJ, Lindenberg S and Lundblad A (1988) Distribution of some Gal B1-24 GlcNAc related carbohydrate antigens on the mouse uterine epithelium in relation to the periimplantation period Journal of Reproductive Immunology 12 297-313

Kimber SJ, White S, Cook $A$ and Illingworth I (1994) The initiation of implantation: parallels between attachment of the embryo and neutrophil-endothelial interaction. In Gamete and Embryo Quality pp 171-178 Ed. L Mastroianni, Jr , HJT Coelingh Bennink, S Suzuki, and HM Vemer. Parthenon, London

Kimber SJ, Illingworth IM and Glasser SR (1995) Expression of carbohydrate antigens in the rat uterus during early pregnancy and after ovariectomy and steroid replacement Joumal of Reproduction and Fertility 130 75-87

Kliman $H$ and Feinberg RF (1990) Human trophoblast-extracellular matrix (ECM) interactions in vitro: ECM thickness modulates morphology and proteolytic activity Proceedings of the National Academy of Sciences USA 87 3057-3061

Koji T, Chedid M, Rubin JS, Slayden Ov D, Csaky KG, Aaronson SA and Brenner RM (1994) Progesterone-dependent expression of keratinocyte growth factor mRNA in stromal cells of the primate endometrium: keratinocyte growth factor as a progestomedin Joumal of Cell Biology 125 393-401

Lafrenie RM and Yamada KM (1996) Integrin-dependent signal transduction Journal of Biological Chemistry 61 543-553

Lessey BA, Damjanovich L, Coutifaris C, Castelbaum A, Albelda SM and Buck CA (1992) Integrin adhesion molecules in the human endometrium. Correlation with the normal and abnormal menstrual cycle journal of Clinical investigation 90 188-195

Lessey BA, llesanmi AO, Lessey MA, Riben M, Harris JE and Chwalisz K (1996) Luminal and glandular endometrial epithelium express integrins differentially throughout the menstrual cycle: implications for implantation, contraception, and infertility American Joumal of Reproductive Immunology 35 195-204

Ligtenberg MJ, Buijs F, Vos HL and Hilkens J (1992) Suppression of cellular aggregation by high levels of episialin Cancer Research 52 2318-2324
Lindenberg S, Sundberg $K$, Kimber SJ and Lundblad A (1988) The milk oligosaccharide, lacto- $N$-fucopentaose 1 , inhibits attachment of mouse blastocysts on endometrial monolayers joumal of Reproduction and Fertility 83 149-158

Liu SW, Tuo W and Bazer FW (1996) Expression of keratinocyte growth factor receptor and mRNA in porcine endometrium Biology of Reproduction 54 (Supplement 1) 75a

Luscinskas FW and Lawler J (1994) Integrins as dynamic regulators of vascular function FASEB journal $8929-938$

Meredith JE, Jr, Winitz S, McArthur Lewis J, Hess S, Ren X-D, Renshaw MW and Schwartz MA (1996) The regulation of growth of intracellular signaling by integrins Endocrine Reviews 17 207-220

Miyamoto S, Teramoto $\mathrm{H}$, Coso OA, Gutkind S, Burbelo PD, Akiyama SK and Yamada KM (1995) Integrin function: molecular hierarchies of cytoskeletal and signaling molecules Joumal of Cell Biology 131 791-805

Pemberton L, Taylor-Papadimitrou J and Gendler SJ (1992) Antibodies to the cytoplasmic domain of Muc-1 mucin show conservation throughout mammals Biochemical and Bioplysical Research Communications 185 167-175

Raab G, Kover K, Paria B, Dey SK and Ezzell RM (1996) Mouse preimplantation blastocysts adhere to cells expressing the transmembrane form of heparin-binding EGF-like growth factor Development 122 637-645

Roberts RM, Trout WE, Mathialagan N, Stallings-Mann $M$ and Ling P (1993) Uterine secretory activity and embryo development Preimplantation Embryo Development, pp 229-243 Ed. BD Bavister. Springer-Verlag, New York

Robertson SA, Seamark RF, Guilbert LJ and Wegmann TG (1994) The role of cytokines in gestation Critical Reviews in Immunology 14 239-292

Samuel CA and Perry JS (1972) The ultrastructure of pig trophoblast transplanted to an ectopic site in the uterine wall Journal of Anatomy 113 139-149

Schwartz MA (1993) Spreading of human endothelial cells on fibronectin or vitronectin triggers elevation of intracellular free calcium Journal of Cell Biology 120 1003-1010

Schwartz MA, Ingber DE, Lawrence M, Springer TA and Lechene C (1991) Multiple integrins share the ability to induce elevation of intracellular pH Experimental Cell Research 195 533-535

Schwartz MA and Denninghoff K (1994) $\alpha$, Integrins mediate the rise in intracellular calcium in endothelial cells on fibronectin even though they play a minor role in adhesion Journal of Biological Chemistry 269 11133-11137

Schwartz MA, Schaller MD and Ginsberg MH (1995) Integrins: emerging paradigms of signal transduction Annual Review of Cell and Dewopmental Biology 11 549-599

Schweighoffer T and Shaw S (1992) Adhesion cascades: diversity through combinatorial strategies Current Opinion in Cell Biology 4824-829

Stewart M, Thiel M and Hogg N (1995) Leukocyte integrins Current Opinion in Cell Biology 7 690-696

Stroband HWJ and Van der Lende T (1990) Embryonic and uterine development during early pregnancy fournal of Reproduction and Ferfility Supplement 40 261-277

Surveyor GA, Gendler AJ, Pemberton L, Das SK, Chakraborty I, Julian J, Pimental RA, Wegner CC, Dey SK and Carson DD (1995) Expression and steroid hormonal control of Muc-1 in the mouse uterus Endocrinology 136 3639-3647

Sutherland AE, Calarco PG and Dansky CH (1993) Developmental regulation of integrin expression at the time of implantation in the mouse embryo Development 119 1175-1186 
Svalander PC, Odin P, Nilsson BO and Obrink B (1990) Expression of cell CAM-105 in the apical surface of rat uterine epithelium is controlled by ovarian steroid hormones Journal of Reproduction and Ferility 88 213-221

Tabibzadeh S (1990) Immunoreactivity of human endometrium: correlation with endometrial dating Fertility and Sterility 54 624-631

Tedder TF, Steeber DA, Chen A and Engel P (1995) The selectins: vascular adhesion molecules FASEB Journal $9866-873$

Tuo $W$ and Bazer FW (1996) Expression of oncofetal fibronectin by porcine conceptuses and uterus throughout gestation Reproduction, Fertility and Development 8 1207-1214

Tuo W, Hamey JP and Bazer FW (1995) Colony-stimulating factor-1 in conceptus and uterine tissues in pigs Biology of Reproduction 53 133-142

Valdizan MC, Julian J and Carson DD (1992) WGA-binding, mucin glycoproteins protect the apical cell surface of mouse uterine epithelium joumal of Cellular Physiology 151 451-465
Wegner CC and Carson DD (1994) Cell adhesion processes in implantation. In Oxford Reviews of Reproductive Biology pp 87-138 Ed. HM Chartion. Oxford University Press, New York

Wesseling J, van der Valk SW, Vos HL, Sonnenberg A and Hilkens J (1995) Episialin (MUC1) overexpression inhibits integrin-mediated cell adhesion to extracellular matrix components Journal of Cell Biology 129 255-265

Whyte A and Robson T (1984) Saccharides localized by fluorescent lectins on trophectoderm and endometrium prior to implantation in pigs, sheep, and equids Placenta 5 533-540

Whyte A and Binns RM (1994) Adhesion molecule expression and infiltrating maternal leucocyte phenotypes during blastocyst implantation in the pig Cell Biology International 18 759-766 